\title{
Microscopic optical model potential based on a Dirac Brueckner Hartree Fock approach and the relevant uncertainty analysis
}

\author{
Ruirui Xu ${ }^{1, \text { a }}$, Zhongyu Ma ${ }^{1}$, Herbert Muether ${ }^{2}$, E.N.E. van Dalen ${ }^{2}$, Tinjin Liu ${ }^{1}$, Yue Zhang ${ }^{1}$, Zhi Zhang ${ }^{1}$, and Yuan Tian ${ }^{2}$ \\ 1 China Nuclear Data Center, China Institute of Atomic Energy, PO Box 275(41), Beijing 102413, China \\ 2 China Institute of Atomic Energy, PO Box 275(41), Beijing 102413, China \\ 3 Institut für Theoretische Physik, Universität Tübingen, Auf der Morgenstelle 14, 72076 Tübingen, Germany
}

\begin{abstract}
A relativistic microscopic optical model potential, named CTOM, for nucleon-nucleus scattering is investigated in the framework of Dirac-Brueckner-Hartree-Fock approach. The microscopic feature of CTOM is guaranteed through rigorously adopting the isospin dependent DBHF calculation within the subtracted T matrix scheme. In order to verify its prediction power, a global study $n, p+$ A scattering are carried out. The predicted scattering observables coincide with experimental data within a good accuracy over a broad range of targets and a large region of energies only with two free items, namely the free-range factor $t$ in the applied improved local density approximation and minor adjustments of the scalar and vector potentials in the low-density region. In addition, to estimate the uncertainty of the theoretical results, the deterministic simple least square approach is preliminarily employed to derive the covariance of predicted angular distributions, which is also briefly contained in this paper.
\end{abstract}

\section{Introduction}

More and more nuclear data for the unstable nuclei are required in the current advanced nuclear applications. Due to scares or absence of experimental data, it is difficult to achieve the predictions for unstable nuclei in a phenomenological way. Optical model potential (OMP) plays an important role in the nuclear reaction process. Therefore, a lot of interests have been attracted in the past to develop a microscopic nucleon-nucleus OMP to predict the total (reaction) cross sections, elastic scattering angular distributions, analysing powers, and spin rotation functions for the applications of astrophysics and the modern reactor design.

Many attempts have been made to derive the microscopic potentials from a realistic $N N$ interaction $[1,2]$. Among them, the potentials derived from the selfenergies in nuclear matter have been successfully applied in the nuclear data study such as the JLM model in a Bruckner Hartree-Fock (BHF) approach by Mahaux et al. [2].

However, it is known that in the relativistic scheme, the spin-orbit potential can be related to the central potential naturally and consistently. Moreover, the saturation properties of symmetric nuclear matter can be well reproduced in the relativistic BHF (DBHF) approach without the three-nucleon force input, which is another good feature to restrict the freedom of potential. As a result, it is appealing to construct a microscopic optical model following the relativistic DBHF method. Some pilot work have been done to explore the possibility of this kind of potential $[3,4]$, and satisfying results are obtained. The potential is named as CTOM to memorize

\footnotetext{
a e-mail: xuruirui@ciae.ac.cn
}

the cooperation between $\boldsymbol{C h i n a}$ Nuclear Data Center and Tübingen University.

The paper is composed as follows. In Sect. 2, the theoretical scheme of CTOM is briefly introduced. Then, the scattering reaction are calculated by CTOM in Sect. 3 for a large mass scale below incident energy $\mathrm{E} \leq$ $200 \mathrm{MeV}$. Currently, two free factors are available in $C T O M$, which indicate the free-range factor $t$ in the applied improved local density approximation (ILDA) and minor adjustments of the scalar and vector potentials in the lowdensity region. A global simulated annealing approach is applied in the optimization for the two factors with the data of $n, p-{ }^{40} \mathrm{Ca},{ }^{208} \mathrm{~Pb}$. All the discussions are presented in Sect. 3. In addition, we also attempt to evaluate the predicted uncertainties of the theoretical results, which are briefly introduced in Sect. 4. Finally, the work is summarized in Sect. 5.

\section{Theoretical scheme of CTOM}

Starting from the realistic $N N$ interactions, the DBHF approach is achieved by considering the equation for two interacting nucleons in nuclear matter. The ladder approximation of the relativistic Bethe-Salpeter (BS) equation is performed, written in an abbreviated operator notation

$$
\mathrm{T}=\mathrm{V}+\text { VQGGT, }
$$

where $\mathrm{T}$ is the nucleon-nucleon interaction matrix in the nuclear medium and $\mathrm{V}$ is the bare $N N$ interaction, respectively. The Pauli Exclusion Principle is included by the $\mathrm{Q}$ operator and the in-medium nucleon propagation of the nucleons is described by the Green's function G. Therefore GG represents the two-nucleon propagator in nuclear matter. The Green's function $G$ fulfils 
the Dyson equation,

$$
\mathrm{G}=\mathrm{G}_{0}+\mathrm{G}_{0} \Sigma \mathrm{G},
$$

$\mathrm{G}_{0}$ denotes the free nucleon propagator, and the selfenergy term $\Sigma$ is defined in first order of the effective interaction $\mathrm{T}$ through the following standard Hartree-Fock equation. In the relativistic scheme, the Lorentz structure of self-energy $\Sigma$ can be expressed as,

$$
\begin{aligned}
& \Sigma^{m}\left(k, k_{F}, \beta\right)=\Sigma_{s}^{m}\left(k, k_{F}, \beta\right)-\gamma_{0} \Sigma_{0}^{m}\left(k, k_{F}, \beta\right)+ \\
& \vec{\gamma} \cdot \vec{k} \Sigma_{v}^{m}\left(k, k_{F}, \beta\right)
\end{aligned}
$$

where $\Sigma_{\mathrm{s}}$ is the scalar part of self-energy, $\Sigma_{0}$ and $\Sigma_{\mathrm{v}}$ denote the time-like and space-like terms of the vector part, respectively. The superscript $\mathrm{m}$ indicates the proton and neutron since they should be distinguished in isospin asymmetric nuclear matter.

It has been a challenge in theoretical nuclear physics for a long time to solve the BHF in a relativistic way, especially for isospin asymmetric nuclear matter. Substantial progress was achieved in recent years through introducing a subtracted T-matrix (STM) representation in the projection technique to solve DBHF strictly in the symmetric and asymmetric nuclear matter [5]. CTOM is developed based on this DBHF solution with the new STM technique.

By solving the Dirac equation, the relativistic Dirac potential for nucleon in nuclear matter can be expressed as:

$$
\begin{aligned}
\mathrm{U}_{s}^{m} & =\left(\Sigma_{s}^{m}-\Sigma_{0}^{m} M\right) /\left(1-\Sigma_{\mathrm{V}}^{m}\right) \\
\mathrm{U}_{0}^{m} & =\left(-\Sigma_{0}^{m}+\varepsilon \Sigma_{\mathrm{V}}^{m}\right) /\left(1+\Sigma_{\mathrm{V}}^{m}\right)
\end{aligned}
$$

and $\varepsilon=\mathrm{E}+\mathrm{M}$ is the single particle energy, $\mathrm{E}$ is the kinetic energy of the nucleon in the free space and $\mathrm{M}$ indicates the mass of the nucleon. It is noticed that the DBHF calculations, however, yield reliable results only for the nuclear matter densities $\rho>0.08 \mathrm{fm}^{-3}$ [4]. In order to compensate the potentials around the low densities $\rho=0.0-0.08 \mathrm{fm}^{-3}$, which correspond to the surface of finite nuclei, the extrapolation of Us and U0 is performed under the constrains of the experimental data of nucleonnucleus scattering. The polynomial fittings are involved to fulfill this task, and the potentials of auxiliary densities at $\rho=0.04 \mathrm{fm}^{-3}$ for the real parts and at $\rho=0.04$ and $0.06 \mathrm{fm}^{-3}$ for the imaginary parts are assumed in the fittings. Meanwhile, we introduce two adjustable factors f1, f2 to slightly modify the 'initial' linear interpolated values at the auxiliary densities. More details of this process are described in Ref. [4].

After eliminating the lower components of the Dirac spinor, the so-called Schrödinger equivalent potentials of finite nuclei, namely $\mathrm{V}_{\text {cent }}, \mathrm{V}_{\mathrm{so}}$ and $\mathrm{V}_{\mathrm{dar}}$, can be obtained. Detailed formula are listed in $[3,4]$. Meanwhile, the improved local density approximation (LDA) is utilized to combine the nucleon distribution in finite nuclei and the Dirac potentials in nuclear matter. Here, 'improved' means a factor $t$ is introduced in LDA to account for a finite-range correction of the nucleon-nucleon interaction.

An optimized system is specially designed to fix the $\mathrm{f} 1$, $\mathrm{f} 2$ in the lower densities and the factor $\mathrm{t}$ in LDA simultaneously. It is proved that the defined $\chi^{2}$ to evaluate the deviation between theoretical values and experimental data becomes better, and the (f1, f2) is fixed as $(0.862,1.142)$, the $t$ value is slightly adjusted from 1.4 to 1.35 for proton-nucleus scattering and 1.45 for neutronnucleus scattering, as shown in [4]. The sensitivities of the parameters are discussed in Sect. 4.

\section{The global analysis of nucleon - nucleus scattering by CTOM potential}

The nucleon density $\rho(\mathrm{r})$ in finite nuclei is the important component in LDA for this potential. The $\rho(\mathrm{r})$ via HartreeFock-Bogoliubov calculation with Gogny D1S force is adopted [7]. As mentioned in Sect. 1, two free factors are expected to fit in the CTOM study. We introduce the global simulated annealing approach to deal with this issue, and the experimental angular distributions of $n, p-{ }^{40} \mathrm{Ca}$ and ${ }^{208} \mathrm{~Pb}$ are used as the restriction. The fitted values can be found in [4]. As a result, the predictions for other stable nuclei are performed using the optimized results with ${ }^{40} \mathrm{Ca}$ and ${ }^{208} \mathrm{~Pb}$. The global comparisons for more nuclei have been carried out. Due to the space limitation, only the angular distributions of elastic scattering of ${ }^{16} \mathrm{O},{ }^{56} \mathrm{Fe}$ and ${ }^{120} \mathrm{Sn}$ are sampled to illuminate the prediction power in Figs. 1-3. The obvious good agreements between the CTOM predictions, experimental data and the phenomenological KD calculation validate the present microscopic potential is reliable in the prediction for scattering reactions. It is deserved to mention that all experimental data plotted in figures can be found in EXFOR library and listed tables in [4].

\section{The statistical analysis and uncertainty evaluation}

In order to assess an entire concept of deviations between the CTOM predictions and experimental data, we have analysed the angular distributions of ${ }^{40} \mathrm{Ca}$ and ${ }^{208} \mathrm{~Pb}$ by using a Gaussian statistical analysis since the two free items are determined under the restriction of these two nuclei. 1029 data points of of ${ }^{40} \mathrm{Ca}$ and ${ }^{208} \mathrm{~Pb}$ in total are incorporated in this analysis listed in [4], and the obtained C/E values and relevant Gaussian fit are shown in Fig. 4. It can be observed that the centre of the fitted Gaussian curve is 0.72 (smaller than 1.0), which means the prediction underestimate the experimental data in the considered energy regions. Actually, the experimental data are generally underestimated by CTOM potentials not only for ${ }^{40} \mathrm{Ca},{ }^{208} \mathrm{~Pb}$ but for other nuclei, as exhibited in Figs. 1-3.

Besides the statistical analysis for the $\mathrm{C} / \mathrm{E}$ values, the deterministic simple least square (SLS) approach [7] is employed to derive the covariance of scattering angular distributions. Normally, the derived covariance $\mathrm{V}_{y}$ in the SLS formula can be briefly shown as:

$$
\mathrm{V}_{\mathrm{y}}=\mathrm{FV}_{\mathrm{C}} \mathrm{F}^{\mathrm{T}} \text {, }
$$

where $\mathrm{F}$ and $\mathrm{F}^{\mathrm{T}}$ represent the sensitivity and the relevant transport matrix of model parameters, and $\mathrm{V}_{c}$ means the covariance of model parameters, which can be calculated by,

$$
\mathrm{V}_{\mathrm{c}}=\left(\mathrm{F}^{\mathrm{T}} \mathrm{V}^{-1} \mathrm{~F}\right)^{-1}
$$

where $\mathrm{V}$ indicates the evaluated covariance of experimental data. In this work, we attempted to combine 

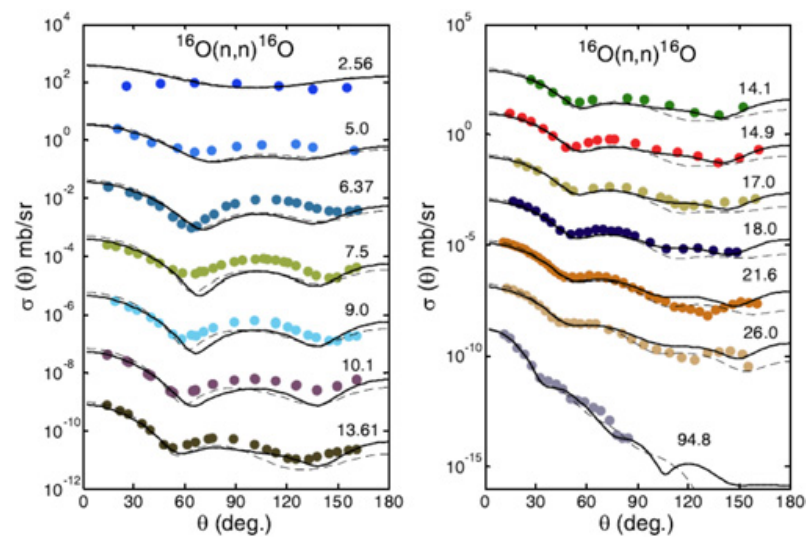

Figure 1. Comparison of predicted differential cross sections by CTOM potential (solid line) and phenomenological KD potentials (dash line) and experimental data (dotted line) for neutron scattered from ${ }^{16} \mathrm{O}$.
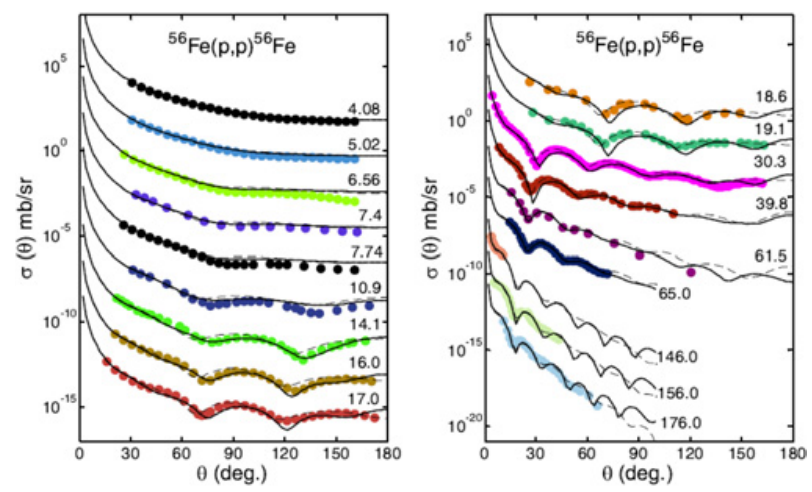

Figure 2. Comparison of predicted differential cross sections by CTOM (solid line) and phenomenological KD potentials (dash line) and experimental data (dotted line) for proton scattered from ${ }^{56} \mathrm{Fe}$.
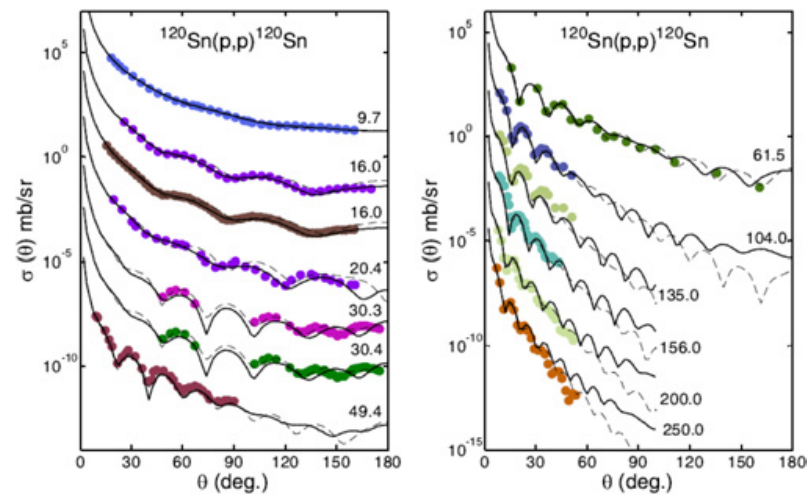

Figure 3. Comparison of predicted differential cross sections by CTOM (solid line) and phenomenological KD potentials (dash line) and experimental data (dotted line) for proton scattered from ${ }^{120} \mathrm{Sn}$.

the uncertainties of theory and measurement to the derived covariance $\mathrm{Vy}$ through the formula above, and a preliminary result is obtained.

Firstly the sensitivities of the range factor $t$ in ILDA at neutron incident energies $2 \mathrm{MeV}$ and $30 \mathrm{MeV}$ are calculated and shown in Fig. 5, and f1 and $\mathrm{f} 2$ for the same energies are shown in Fig. 6. The sensitivities increase with the increasing energy both for real and imaginary parts, as shown in these figures.

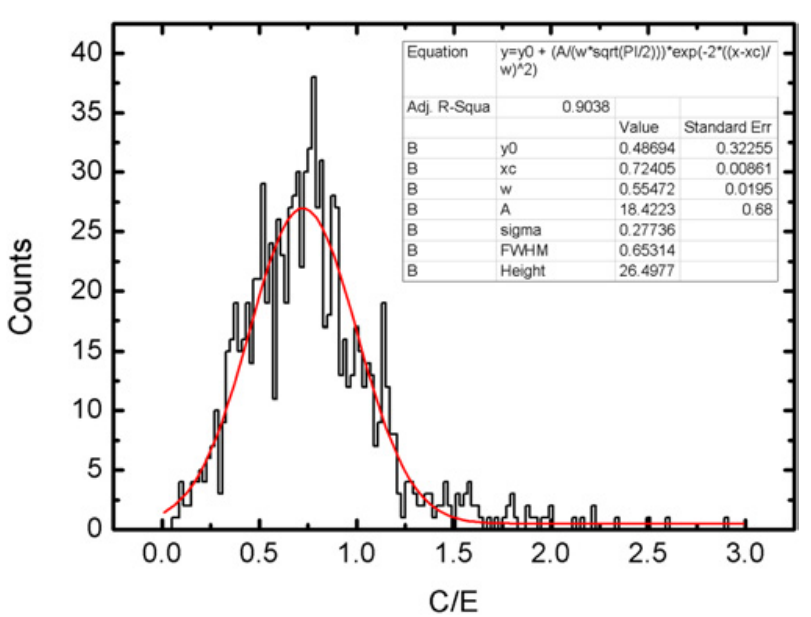

Figure 4. Gaussian analysis for the $\mathrm{C} / \mathrm{E}$ values of $\mathrm{n}-{ }^{40} \mathrm{Ca},{ }^{208} \mathrm{~Pb}$.
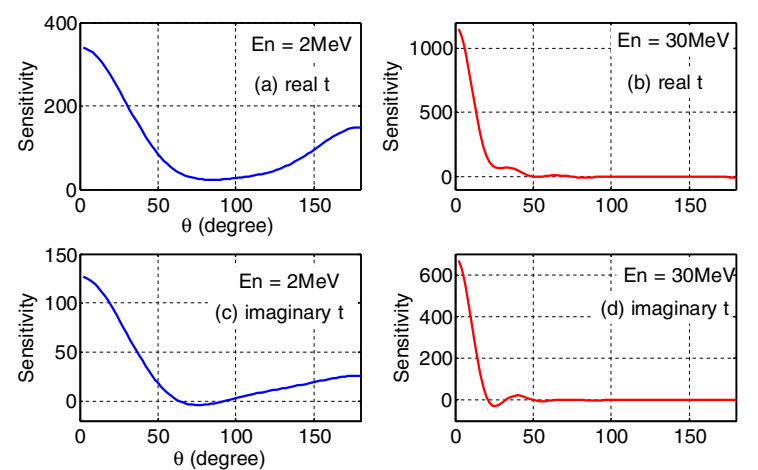

Figure 5. The sensitivities of the range factor $\mathrm{t}$ at $\mathrm{E}_{n}=2 \mathrm{MeV}$ and $30 \mathrm{MeV}$.
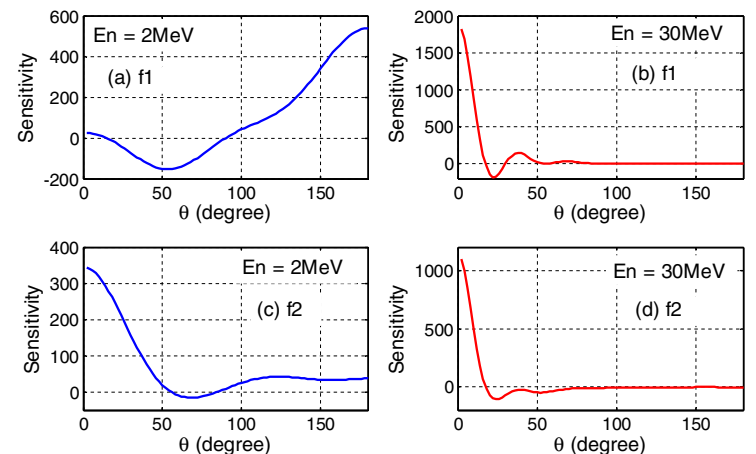

Figure 6. The sensitivities of the factors $\mathrm{f}_{1}$ and $\mathrm{f}_{2}$ at $\mathrm{E}_{n}=2 \mathrm{MeV}$ and $30 \mathrm{MeV}$.

The experimental covariance matrix $\mathrm{V}$, as shown in Eq. (6), is an important input in SLS. In CTOM, the uncertainty of experimental data comes from the measurements of ${ }^{40} \mathrm{Ca}$ and ${ }^{208} \mathrm{~Pb}$, especially the correlation between the data of two nuclei. After systematically looking through the common conditions of current experimental data of ${ }^{40} \mathrm{Ca}$ and ${ }^{208} \mathrm{~Pb}$, we roughly estimate the experimental statistic uncertainty is $10 \%$, the correlated uncertainty between different angles at the same energy is $3 \%$ with the assumed correlated coefficient 0.5 ; besides, we assume an additional correlated uncertainty about $15 \%$ not included in experimental data with the assumed correlated coefficient 0.3 . It should be noted that the present experimental covariance is constructed based 
Table 1. The derived Rho matrix of free parameters.

\begin{tabular}{|c|c|c|c|c|}
\hline & Real t & Imaginary t & $\mathrm{f} 1$ & $\mathrm{f} 2$ \\
\hline Real t & 1 & -0.204 & -0.077 & 0.157 \\
\hline Imaginary t & -0.204 & 1 & -0.627 & -0.347 \\
\hline f1 & -0.077 & -0.627 & 1 & 0.362 \\
\hline f2 & 0.157 & -0.347 & 0.362 & 1 \\
\hline
\end{tabular}

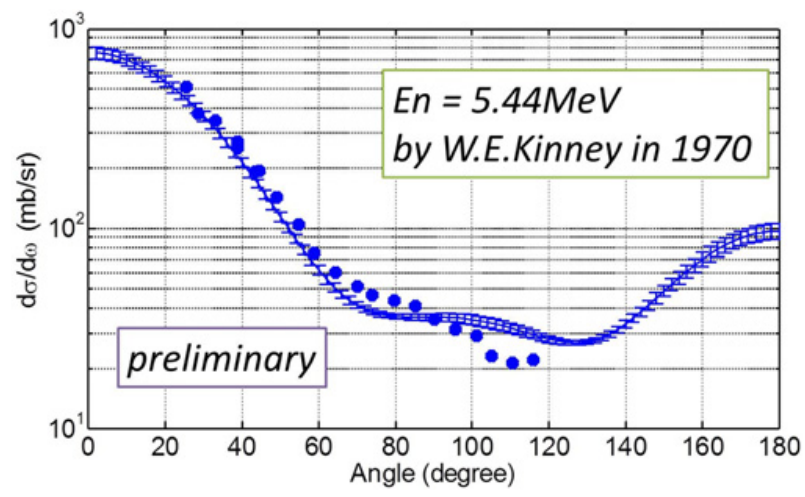

Figure 7. The comparison between our predicted centre value of $n+{ }^{27} \mathrm{Al}$ at $\mathrm{En}=5.44$, and the measurement was reported by W. Kinney in 1970. The uncertainty from SLS is also presented.

on the common sense information and assumed values above. More accurate evaluation is desired in the future work.

By combining the sensitivity and experimental uncertainty in SLS, the covariance of free parameters $V c$ is obtained. The free parameters (real t, imaginary t, f1, f2) with the resultant standard deviations are expressed as: Real $\mathrm{t}=1.45 \pm 0.01$; Imaginary $\mathrm{t}=1.45 \pm 0.047$; $\mathrm{f} 1=0.862 \pm 0.007 ; \mathrm{f} 2=1.142 \pm 0.012$, and the derived correlated coefficient of parameters, which is defined as

$$
\operatorname{Rho}(i, j)=\operatorname{Vc}(i, j) / \operatorname{sqrt}(\operatorname{Vc}(i, i), \operatorname{Vc}(j, j)),
$$

where $\mathrm{i}$ and $\mathrm{j}$ indicate different parameters, are shown in Table 1. The covariance of the CTOM predictions can be obtained based on $V c$. As an example, the predicted angular distribution of $\mathrm{n}+{ }^{27} \mathrm{Al}$ at $\mathrm{En}=5.44 \mathrm{MeV}$ with the evaluated uncertainty is plotted in Fig. 7. Due to our rough assumptions of uncertainties and correlations, the current uncertainty by SLS looks still underestimated, it could be considered as a minimum estimation now.

\section{Summary}

A microscopic nucleon-nucleus optical potential, CTOM is developed recently to describe the nucleon scattering data for stable nuclei. CTOM is founded in the microscopic isospin-dependent DBHF theory with very few free parameters, which provides more confidence in the application of the prediction for unstable nuclei in the near future. The performance of CTOM is sampled by ${ }^{16} \mathrm{O}$, ${ }^{56} \mathrm{Fe}$ and ${ }^{120} \mathrm{Sn}$ at the nucleon incident energies from 1 to $200 \mathrm{MeV}$ in this work. Good agreement between the predictions and experimental data verify its feasibility. Certainly, it is impossible to depict all quantities perfectly only using the present spherical nuclear MOP such as for the strongly deformed nuclei around rare earth and actinide regions [4]. Many other physics mechanisms e.g., the particle-vibration coupling, are expected to compensate CTOM.

In order to evaluate the uncertainty of predictions, the SLS approach is introduced to derive the covariance of theoretical results. After making some assumptions for the covariance of experimental data, we preliminarily derive the covariance of our predicted values. Currently, because the reliability of assumed uncertainty and correlation is not good yet, the derived uncertainty looks underestimated, which require further elaborate evaluation to experimental data of ${ }^{40} \mathrm{Ca}$ and ${ }^{208} \mathrm{~Pb}$ in future.

The authors thank Prof. S. Hilaire for kindly supplying us all the density distributions of finite nuclei. This work has been supported by the National Natural Science Foundation of China (Grants No. U1630143, No. 11305270, No. 11275018); the Deutsche Forschungsgemeinschaft (DFG) under Contract No. $\mathrm{Mu} 705 / 10-1$.

\section{References}

[1] K. Amos, P.J. Dortmans, H.V. von Geramb, S. Karataglidis, J. Raynal, Adv. Nucl. Phys. 25, 1 (2000)

[2] J.P. Jeukenne, A. Lejeune, and C. Mahaux, Phys. Rev. C 16, 80 (1977)

[3] R. Xu, Z. Ma, E.V.E. van Dalen, and H. Muetrher, Phys. Rev. C 034613, 85 (2012)

[4] R. Xu, Z. Ma, Y. Zhang, Y. Tian, E.V.E. van Dalen, and H. Muetrher, Phys. Rev. C 94, 034606 (2016)

[5] E.N.E. van Dalen, C. Fuchs, and A. Faessler, Nucl. Phys. A 744, 227 (2004)

[6] S. Hilaire, Eur. Phys. J. A 33, 233 (2007)

[7] D. Smith, ANL/NDM-128 (1993) 